\title{
Electrical Power Peaks in the Simulation of Material Handling Systems
}

\author{
Karsten Turek ${ }^{*}$, Armin Siegel, Thorsten Schmidt \\ Inst. of Material Handling and Industrial Engineering, Technische Universität Dresden, 01062 Dresden, Germany \\ *karsten.turek@tu-dresden.de
}

SNE 30(4), 2020, 189-195, DOI: 10.11128/sne.30.tn.10539 Received: July 31, 2020 (Selected ASIM SPL 2019 Postconf. Publ.); Revised: Oct. 8, 2020; Accepted: Oct. 20, 2020

SNE - Simulation Notes Europe, ARGESIM Publisher Vienna ISSN Print 2305-9974, Online 2306-0271, www.sne-journal.org

Abstract. The expansion of local power supply with renewable sources is one option for shaping the energy transition. Power supply from renewable sources has to be balanced with power demand of dynamic technical systems. Investigation of detailed electrical power demands in production and logistics systems receives increasing attention in this context. This paper describes a method for the analysis of power demand of material handling components within a material flow simulation. The simulation approach enables the examination of power peaks as well as the system throughput during system operations. The power calculation model was developed with the environment MATLAB and integrated into the AutoMod simulation environment. The simulation of a high-bay warehouse with several handling machines delivers the proof of concept. We introduce a new control approach for the reduction of power peaks and investigate its effect on the transport performance with simulation experiments. The results of experiments show that the control approach can limit power peaks without a considerable reduction of the transport performance in the warehouse system. The achieved reductions of the maximum power peaks range from $80 \%$ up to $67 \%$ in different settings and correspond with a transport performance decrease of no more than $3 \%$ in maximum.

\section{Introduction}

The consideration of energy demand and energy efficiency takes place in planning, design, and control of technical systems and processes over the last two decades.
In addition to the monetary aspects of energy saving, legal requirements force increased efforts by industry to protect the environment and the climate.

Accordingly, energy and environmental aspects received greater attention in simulation of production and logistics systems, as [1] show in their publication. To include these new aspects, various approaches and methods extended discrete event simulation, which is an established tool for analysis of these systems.

Mainly aspects of long-term energy saving and energy efficiency dominate the studies. The investigation of short-term electrical power peaks is a further topic worth to consider in technical systems. The following paragraphs motivate this.

Renewable energy sources, such as wind and sun, provide a volatile energy supply due to technological reasons. The compensation of asynchronous fluctuations of energy supply and energy demand is complex. Energy storage systems may compensate short-term peaks. The planning and control of suitable storage systems requires knowledge about power peaks that occur in the technical system.

A further motivation results from the dimensioning of electrical supply systems (e.g., transformers, circuit breakers) that depends on the estimated maximum power in the electrical subnetwork. In today's practice, the lack of methods for predicting power peaks leads to an oversized design of supply systems [2]. A limitation of the peaks enables a reduction in size and cost of the electrical supply components.

This article considers the analysis of the electrical power demand of material handling systems by means of discrete event simulation. We discuss representations of energy-related aspects in calculation models and ways of integrating power calculation into material flow simulation. We explain our selected implementation. Simulation experiments evaluate a control intervention for reducing the power peaks in a high-bay warehouse system. 


\section{Related Work}

The consideration of energy aspects in the simulation of production and logistics systems relates to two topics:

1. The representation of energy-related aspects in calculation models.

2. The integration of energy calculation models into simulation.

In advance to a simulation study, the energy-related aspects of real technical systems must be mapped into calculation models. For this topic, the following introduction of related work focuses on energy models of storage and retrieval machines (SRMs) in high-bay warehouses. The second part of this chapter discusses different ways of integrating energy calculation models into discrete event simulation (DES).

\subsection{SRM Energy Models}

Automatic SRMs used in high-bay warehouses receive special attention in the energy analysis of material handling systems. Due to their construction and mode of operation, these devices have a high energy demand per transport as well as significant power peaks when starting up. SRM serve horizontally- and vertically-arranged storage locations in a warehouse storage aisle. The machines are equipped with a drive for horizontal movement and another drive for vertical movement. Industrial storage systems typically consist of multiple parallel aisles.

Several studies, such as [3], [4], and [5], investigated the factors influencing the energy demand of SRMs. The focus of these studies lies on the development and validation of calculation models for energy demand. The aim is to determine the energy requirement of SRM with different configurations or to select the most energy-efficient travel trajectory for a transport in advance. The energy demand calculations in these studies are based on the same motion transformation model approach and differ only in details. In addition, models of this type enable in principle the identification of power peaks.

The model equations used for this paper are based on the author's previous work and explained in detail in [3] and [14]. The approach in general is briefly described later in Section 2.1.

Hahn-Woernle considers in [2] the overlap of power demand and its reduction by delaying the start of SRM transport orders. The warehouse system is represented as a simulation model in MATLAB/Simulink.
The strengths of the model lie in the detailed mapping of the power demand. The approach is limited to the singular consideration of storage systems and is not suitable for the simulation of interactions within a material flow system.

Shifting the starting times of the drives can minimise the maximum power demand at simultaneous start of SRMs [6]. The authors use an optimization with a genetic algorithm to determine the best shifting. The approach focuses on the single situation that all SRMs start at the same time. The authors do not explain the application in case of constantly new transport orders.

\subsection{Energy-related Simulation}

The representation of energetic interrelationships of plants and processes in DES can be differentiated in terms of their complexity in a modelling by

a) constant power values in discrete time periods, assigned to plant states or process phases, or

b) continuous variable power values derived from physical models or measurement series.

Roemer and Strassburger stated in their overview that the majority of publications use a discrete event system model with state-based energy representation [7]. Only a few publications describe an approach with representation (b), among them [10] and [11].

The modelling with phase-wise constant power values enables direct integration into discrete event simulation, if the phase changes can be represented by events. The power value is fixed from event to event. This implementation offers sufficient accuracy for a calculation of the energy demand in a simulation study, but not for an analysis of power curve and power peaks [8].

Modelling variable power values offers higher accuracy in the mapping of real electrical power demand curves, but also causes higher efforts for data acquisition, model generation, and validation.

The application of option (b) in material flow simulation requires a combination of the event-based model of the material handling system with a time-continuous model of the power demand. There are three basic approaches to this (cf. [9]):

1. Simulation with a downstream energy analysis with external software.

2. Dynamic coupling of the simulation with a parallel energy consideration in an external tool (cf. [10]).

3. Integration of energy analysis and simulation in one application (cf. [11] and [12]). 
The downstream approach is sufficient for a static system analysis, but does not allow for any dynamic influence on the simulated process and, therefore, it is not suitable for analysis of strategies to reduce power peaks.

The combination of different tools offers an advantage by using problem-specific modelling domains. The dynamic coupling enables model interaction. The main disadvantage of this option is the additional effort for the model coupling and synchronization.

The integration of the energy calculation and the operations simulation in a uniform application avoids the disadvantages of the previous options, whereby [13] emphasize in their evaluation that the linkage represents a considerable challenge.

This paper describes an approach that uses two different tools for modelling. A discrete event model describes the transport operations of the material handling system. A continuous value model calculates the power demand curve of material handling components. The continuous value model is integrated in the DES environment.

\section{Simulation Model}

We base our investigation of electrical power peaks on a typical pallet high-bay warehouse. The use case was modelled using the simulation environment AutoMod, which supports the mapping of various material handling subsystems by means of ready-made modules and predefined simulation routines.

The geometric representation of the high-bay warehouse in the simulation depends on the number of aisles, vertical levels, and storage bays per level. The SRM gets the target position in the shelf front at random or specified by program code. The transport time results from parameterisable kinematic parameters like speed and acceleration as well as from additional times like load pick-up time and load set-down time.

\subsection{SRM Power Model}

This paper describes an approach that corresponds with option (b) from Section 1.2 using continuous power values. This option enables a fine calculation of the power demand in time and thus of the power peaks of the SRM.

The physical energy-related model for determining the power demand is already described in detail in [14]. Basis is a kinematic motion model for the chassis and for the lifting system. A 7-phase motion model arises by the use of a constant jerk in the motion equations (Figure 1).
The other movement variables (acceleration, velocity, and distance) result accordingly from its integration. The combination of motion variables and moving masses determines the mechanical power demand.

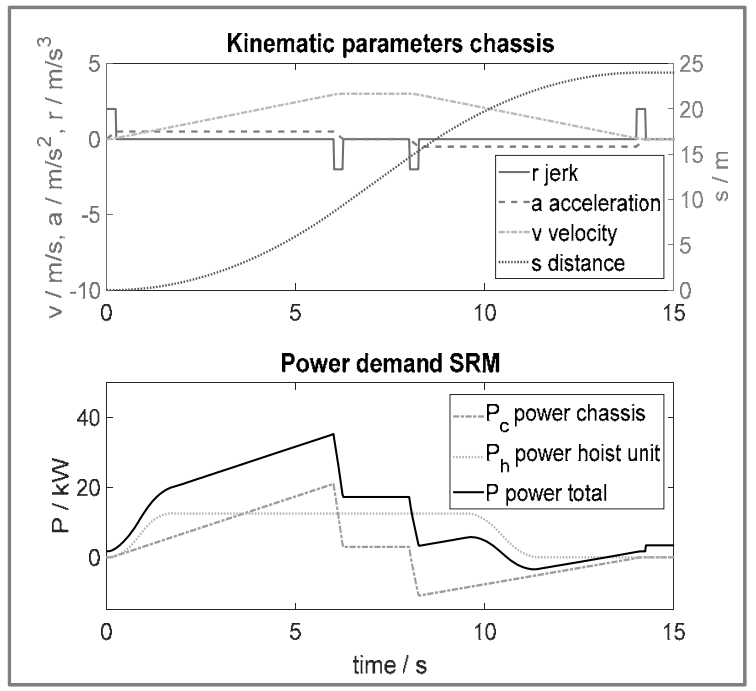

Figure 1: SRM power demand model.

Mechanical losses (e.g., the driving resistance of the wheel-rail combination) and electrical losses (e.g., in the frequency converter) of the power transmission are included in the calculation as well as the efficiency of the drives.

The power demand for the complete motion sequence of an SRM results from the superposition of horizontal and vertical motion (cf. Figure 1). The power demand of the load handling device is considerably lower and can, therefore, be simplified by using constant values.

The used energy-related model was developed in a former research project and calibrated by power measurement data of a real SRM, described in [3].

Table 1 gives an overview of power key values of the SRM drives in the later experiments.

\begin{tabular}{lc}
\hline & $\begin{array}{c}\text { Max. power } \\
\text { in kW }\end{array}$ \\
\hline Horizontal drive start up & 20.0 \\
\hline Horizontal drive continues & 4.0 \\
\hline Lifting drive & 16.0 \\
\hline Load handling device & 2.0 \\
\hline
\end{tabular}

Table 1: Power values of the modelled SRM. 


\subsection{Power Model Implementation}

There are different ways to connect software systems. The coupling options include the use of DDE (Dynamic Data Exchange), OPC (Open Platform Communications), TCP/IP (Transmission Control Protocol/Internet Protocol), and DLL (Dynamic Link Library). A DLL can integrate an encapsulated software code into another program. This solution supports our hybrid modelling approach. It works without additional tool communication interfaces and offers a high data transmission rate. For these reasons we selected the DLL technology.

The MATLAB environment offers the possibility to compile developed functions to a DLL using the MATLAB Coder. The restriction that not all built-in functions of MATLAB are supported is not limiting the selected model context. The calculation equations of the energy-motion model were developed in MATLAB and then transferred to a DLL. The DLL is supplemented by functions for setting parameters of the calculation model and for communicating with external software. The Cinterface of the AutoMod simulation system supports the integration of DLL into simulation models. It requires additional header files and the declaration of the utilized DLL functions within the specific model.

At the beginning of an experiment, the simulation model initializes the DLL with the technical parameters of the SRMs. During simulation, the model sends start time, start location, and destination of each SRM trip to the DLL and receives the calculation results for further control decisions. The DLL calculates the movement trajectory of the SRM and the corresponding power demand for the complete trip in advance. Based on the saved data of previous trips, it calculates the total power of the warehouse including the new trip. The identification of power peaks is inclusive.

As a side effect, the DLL can calculate the travel time more precisely than the built-in function in the simulation system that uses a 3-phase motion model only. An additional wait for synchronization option in the simulation model supports the use of this returned travel time value.

\section{Power Peak Limitation}

The next steps after identification are reduction and limitation of power peaks. The consideration that the system power peaks are primarily caused by the superimposition of start-up processes of SRMs supports the selected approach. The significantly highest power demand is in the short phase of accelerating the SRM drives.
Following the approach in [2], slight shifts in the start-up processes can avoid their temporal overlap and reduce system power peaks.

A control procedure for reducing and limiting the power peaks in a high-bay warehouse system complements the previously presented implementation. It was developed as part of a funded research project.

Control approach. A central control for start shifting was implemented in the simulation model with two different sets of restrictions:

a) Start-up restriction: The number of simultaneous start processes is limited by the control.

b) Power restriction: A given limit of the maximum power demand is monitored by the control.

By the start-up restriction, the control monitors acceleration and velocity of the SRMs. As they are responsible for high power peaks, we exclusively consider trips where starting and lifting take place simultaneously. A new trip is delayed as long as the SRM start-up limit is reached. The limitation of simultaneous starting processes offers a limitation of the maximum power requirement.

In power restriction, the control monitors the total power demand of the system. Before starting the SRM, each new trip adds virtually its predicted power demand to the current system demand to test the power limit. If the specified power maximum is exceeded, the start time of the new trip will be shifted with step size one second and the new maximum power is calculated again. The control executes shifting until the system power maximum stays below the limit or the maximum possible delay is reached. The maximum possible delay is a selectable starting parameter for experiments.

Finally, the DLL returns the calculated start delay of the next trip to the simulation model. The effect of this control measure on the reduction of power peaks and simultaneously on the transport performance in the system was focussed in the following experiments.

\section{Experiments and Results}

The high-bay warehouse used as model case for the experiments in this paper consists of eight aisles with ten levels and one SRM per aisle. This configuration corresponds to a real-system pallet warehouse in terms of size. The AutoMod simulation subsystem AS/RS enables a detailed representation of real-system properties. 
The model includes the following assumptions: Storage and retrieval of the pallets are independent processes. The throughput target value is the number of pallets per aisle per hour. A random selection sets the storage position in an aisle regardless of the fill status. A dedicated storage bin management is not taken into account.

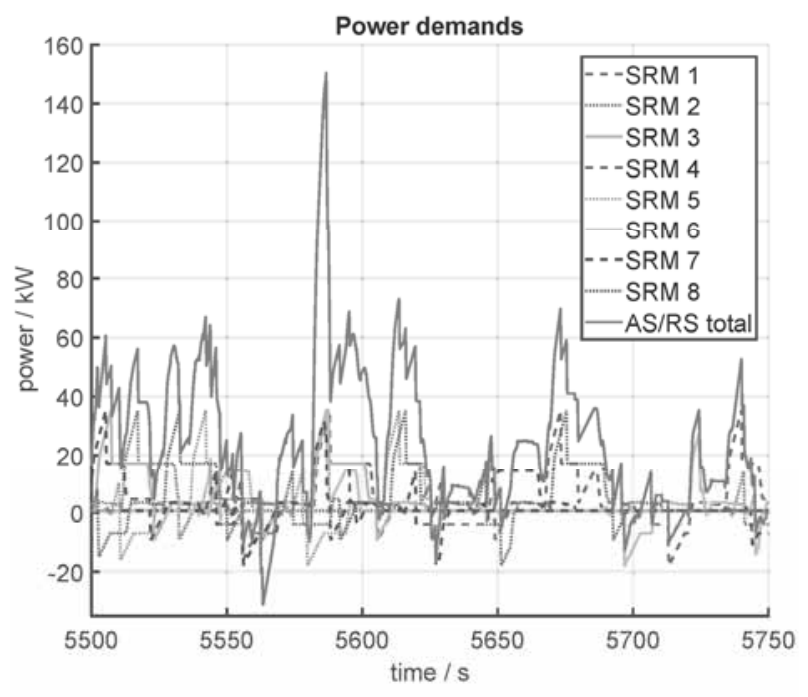

Figure 2: Example of total power demand for eight SRMs.

Table 2 defines the kinematic parameters of the SRM. Load pickup time and load set-down time are twelve seconds each. Each experiment lasted ten hours of simulation time. The evaluation of the control approach is based on two different transport scenarios:

1) Unidirectional transports: Maximum throughput with only storage trips

2) Bidirectional transports: Maximum throughput with storage and retrieval trips.

\begin{tabular}{lcc}
\hline & Horizontal & Lift \\
\hline Velocity $[\mathrm{m} / \mathrm{s}]$ & 2.0 & 1.0 \\
\hline Acceleration $\left[\mathrm{m} / \mathrm{s}^{2}\right]$ & 0.5 & 1.0 \\
\hline Jerk $\left[\mathrm{m} / \mathrm{s}^{3}\right]$ & 2.0 & 1.2 \\
\hline
\end{tabular}

Table 2: Kinematic parameters of SRM.

In Scenario 1, each SRM achieves about 55 storage operations per hour in case of no peak limitations. The SRM transports a load from the transfer point to a randomly selected storage location. After completion of the transport operation, the SRM returns empty to the transfer point and collects the next load immediately. This procedure results in 110 trips per hour.
In Scenario 2, each SRM fulfils about 34 storage and 34 retrieval operations. First, the SRM transports a load from the transfer point into the shelf. Then it travels empty to the start location for the retrieval transport from the shelf. Finally, it returns the retrieval load to the transfer point in front of the shelf. This creates a double cycle with three movements. In case of maximum utilization only double cycles occur. This results in a total of about 100 trips per SRM and hour in the experiment.

Both scenarios were simulated with both control options. The system power limits in the second approach were $120 \mathrm{~kW}$ and $140 \mathrm{~kW}$. The maximum allowed shift limit is twelve seconds in all experiments.

\begin{tabular}{ccccc}
\hline $\begin{array}{c}\text { Control } \\
\text { limit }\end{array}$ & $\begin{array}{c}\text { Shift in s } \\
\text { per SRM } \\
\text { per hour }\end{array}$ & $\begin{array}{c}\text { Peak } \\
\text { power } \\
\text { in kW }\end{array}$ & $\begin{array}{c}\text { Through- } \\
\text { put } \\
\text { per hour }\end{array}$ & $\begin{array}{c}\text { Through- } \\
\text { put } \\
\text { in \% }\end{array}$ \\
\hline 1 SRM & 106 & 121 & 53.3 & 97 \\
\hline 2 SRM & 10 & 146 & 54.9 & 99 \\
\hline 3 SRM & 1 & 165 & 55.1 & 100 \\
\hline No limit & -- & 178 & 55.1 & 100 \\
\hline 120 kW & 3 & 119 & 55.0 & 100 \\
\hline 140 kW & 1 & 139 & 55.1 & 100 \\
\hline
\end{tabular}

Table 3: Results for Scenario 1.

Limiting the number of devices starting simultaneously does not support a precise limit of power peaks. It only avoids the unfavourable superposition of single peaks of the SRMs' start-up processes. The remaining motion processes continue to contribute to the power demand of the system. Furthermore, start delays can also occur in situations where they are not necessary to reduce peak loads. Not every start-up superposition leads to a relevant power peak.

On the basis of a theoretical maximum of eight parallel start-up processes in the example system, a limitation to two start-up processes leads to a significant reduction in the power peaks by $80 \%$ compared to the unlimited approach. The throughput of the system drops by only $1 \%$. It should be noted that superposition of eight unfavourable start-up processes is an extremely rare event.

The approach with a fixed limit as in the power restriction control enables a much better adapted response to the current demand situation. The extent of the start postponements is considerably smaller with an equivalent reduction of the power peaks. The throughput of the system remains almost unchanged even with the limit of $120 \mathrm{~kW}$. 


\begin{tabular}{ccccc}
\hline $\begin{array}{c}\text { Control } \\
\text { limit }\end{array}$ & $\begin{array}{c}\text { Shift in s } \\
\text { per SRM } \\
\text { per hour }\end{array}$ & $\begin{array}{c}\text { Peak } \\
\text { power } \\
\text { in kW }\end{array}$ & $\begin{array}{c}\text { Through- } \\
\text { put } \\
\text { per hour }\end{array}$ & $\begin{array}{c}\text { Through- } \\
\text { put } \\
\text { in \% }\end{array}$ \\
\hline 1 SRM & 96 & 108 & 66.2 & 97 \\
\hline 2 SRM & 6 & 128 & 67.9 & 99 \\
\hline 3 SRM & 1 & 155 & 68.1 & 100 \\
\hline No limit & -- & 160 & 68.2 & 100 \\
\hline 120 kW & 2 & 119 & 68.1 & 100 \\
\hline 140 kW & 0 & 139 & 68.2 & 100 \\
\hline
\end{tabular}

Table 4: Results for Scenario 2.

Both control options have the need for a higher-level coordination between the driving controls of the SRMs in common.

The control with start-up restriction focusses on the SRM start processes in the warehouse system. This implementation does not require a detailed model or measurement of the SRM power demand. It is based on the assumption that the start-up process produces the highest power peaks.

The control with power restriction permanently monitors power demand of the system. It needs a power forecast model. On the one hand, this implementation requires more effort for this reason. On the other hand, it can guarantee considerably lower maximum power peaks accompanied by better system throughput.

To summarize for a practical implementation, limiting the number of devices that start simultaneously is technically a more realistic option than monitoring the power demand of the system. However, even this option requires changes in the current system control structure. The individual SRM controls need to communicate with an additional central coordination instance.

The experiments were carried out with the technical maximum throughput of the SRMs. In these cases start delays always lead to a reduction in throughput. In the vast majority of practical applications, the logistic system's throughput is lower. There are idle phases between transport orders. Therefore, short start delays will have a significantly lower impact on the throughput of the system than in the presented scenarios. We suppose that power peak limitation in real systems can be reached without a reduction in throughput.

\section{Summary and Outlook}

This publication presents an enhancement of material flow simulation by a calculation of electrical power demand in order to evaluate systems power peaks.

First, we motivated the aim of knowing and limiting power peaks in material handling systems. Second, we set the chosen integration approach in relation to previous work in the field. Then, we explained the way of modelling and the implementation. Finally, we used the simulation of a high-bay warehouse with several SRMs to investigate the extent of power peak limitation in relation to the transport performance.

We applied a hybrid modelling approach to create the simulation model. The discrete event material flow model of a high-bay warehouse system was implemented in the AutoMod simulation environment without power calculation. In parallel, the power calculation model was developed with the MATLAB environment. Subsequently, the calculation model was compiled into a DLL and in this form integrated into the simulation model. Based on the parameters of the SRM, the calculation model evaluates the transport order data given from simulation and predicts the power demand for the warehouse system.

Furthermore, we presented a control approach that uses a device start delay for the reduction of system power peaks. In simulation experiments we investigated two different power restrictions and their effect on the transport performance. The results show that both approaches can effectively limit power peaks in combination with a small reduction in the transport performance of the warehouse system. The implementation and test of the control in a real-world system are the next logical steps. The modelling approach and the way of implementation are not limited to warehouse systems.

The idea of using electrical energy storages in material handling systems motivates further work in the field. In a volatile electrical demand situation, energy storage systems can cut the unwanted peaks and lower the load on the power network. Energy storage systems act as a buffer between supply and demand, enabling the integration of local renewable energy sources in the power supply. The extension of the material flow simulation by models and control algorithms of energy storage systems supports the research in this development. 


\section{Acknowledgement}

This research project IGF 19324 BR from the Research Association Intralogistics/Materials Handling and Logistics Systems (IFL) was funded by the German Federation of Industrial Research Associations (AiF) within the programme for promoting the Industrial Cooperative Research (IGF) of the German Ministry of Economic Affairs and Energy (BMWi) on the basis of a resolution of the German Bundestag.

\section{References}

[1] Wenzel S, Peter T, Stoldt J, Schlegel A, Uhlig T, Josvai J. Considering energy in the simulation of manufacturing systems. In: Rabe M, Juan AA, Mustafee N, Skoogh A, Jain S, Johansson B, editors. Proceedings of the 2018 Winter Simulation Conference. Piscataway, NJ: IEEE; 2018. 3275-3286. doi: $10.5555 / 3320516.3320904$

[2] Hahn-Woernle P, Günthner WA. Power-load management reduces energy-dependent costs of multi-aisle mini-load automated storage and retrieval systems. International Journal of Production Research. 2018; 56(3): 1269-1285. doi: 10.1080/00207543.2017.1395487

[3] Siegel A, Schulz R, Turek K, Schmidt T, Zadek H. Modeling the energy need of storage and retrieval vehicles and different storage operating strategies for the reduction of the energy need. Logistics Journal. 2013; 1-18. doi: 10.2195/lj_Proc_siegel_de_201310_01

[4] Ertl R. Energiebedarfsermittlung und Energieeffizienzbewertung von Regalbediengeräten in automatischen Kleinteilelagern. Munich, Germany: Technical University of Munich; 2016.

[5] Braun MSA. Entwicklung, Analyse und Evaluation von Modellen zur Ermittlung des Energiebedarfs von Regalbediengeräten. Karlsruhe, Germany: Karlsruhe Institute for Technology; 2016.

[6] Cardenas JJ, Garcia A, Romeral JL, Andrade F. A Genetic algorithm approach to optimization of power peaks in an automated warehouse. $35^{\text {th }}$ Annual Conference of IEEE Industrial Electronics; 2009 November; Porto, Portugal. 3297-3302.

doi:10.1109/iecon12502.2009
[7] Roemer AC, Strassburger S. A review of literature on simulation-based optimization of the energy efficiency in production. In: Roeder TMK, Frazier PI, Szechtman R, Zhou E, Huschka T, Chick SE, editors. Proceedings of the 2016 Winter Simulation Conference. Piscataway, NJ: IEEE; 2016. 1416-1427. doi: 10.1109/WSC.2016.7822194.

[8] Roemer AC, Rueckbrod M, Strassburger S. Eignung kombinierter Simulation zur Darstellung energetischer Aspekte in der Produktionssimulation. In: Deatcu C, Schramm T, Zobel K, editors. Tagungsband ASIM SST 2018 - 24. Symposium Simulationstechnik; 2018 October, Hamburg, Germany. Vienna, Austria: ARGESIM. 73-80. doi: 10.11128/arep.56.

[9] Herrmann C, Thiede S, Kara S, Hesselbach J. Energy oriented simulation of manufacturing systems - Concept and application. CIRPAnnals. 2011; 60(1): 45-48. doi: 10.1016/j.cirp.2011.03.127.

[10] Peter T, Wenzel S. Simulation-based planning and evaluation of energy efficiency for production systems in car manufacturing. In: Rabe M, Clausen U, editors. Simulation in Production and Logistics. Stuttgart: Fraunhofer Verlag; 2015. 535-544.

[11] Stoldt J, Schlegel A, Putz M. Enhanced integration of energy-related considerations in discrete event simulation for manufacturing applications. Journal of Simulation. 2016; 10(2): 113-122. doi: 10.1057/jos.2015.24

[12] Schmidt A, Pawletta T. Hybride Modellierung fertigungstechnischer Prozessketten mit Energieaspekten in einer ereignisdiskreten Simulationsumgebung. In: Wittmann J, Deatcu C, editors. Tagungsband ASIM SST 2014 - 22. Symposium Simulationstechnik; 2014 September; Berlin, Germany. Vienna, Austria: ARGESIM. 109-116. doi: 10.11128/arep.52

[13] Garwood TL, Hughes BR, Oates MR, O'Connor D, Hughes R. A review of energy simulation tools for the manufacturing sector. Renewable and Sustainable Energy Reviews. 2018; 81(1): 895-911. doi: 10.1016/j.rser.2017.08.063

[14] Siegel A, Turek K, Michelini E, Schmidt T. Hybrid modeling approach for prediction of energy demand and power peaks in intralogistic systems. In: Deatcu C, Schramm T, Zobel K, editors. Tagungsband ASIM SST 2018 - 24. Symposium Simulationstechnik; 2018 October, Hamburg, Germany. Vienna, Austria: ARGESIM. 81-88. 